\title{
Systematic Reviews in Educational Research: Methodology, Perspectives and Application
}

\author{
Mark Newman and David Gough
}

\section{$1 \quad$ What Are Systematic Reviews?}

A literature review is a scholarly paper which provides an overview of current knowledge about a topic. It will typically include substantive findings, as well as theoretical and methodological contributions to a particular topic (Hart 2018, p. xiii). Traditionally in education 'reviewing the literature' and 'doing research' have been viewed as distinct activities. Consider the standard format of research proposals, which usually have some kind of 'review' of existing knowledge presented distinctly from the methods of the proposed new primary research. However, both reviews and research are undertaken in order to find things out. Reviews to find out what is already known from pre-existing research about a phenomena, subject or topic; new primary research to provide answers to questions about which existing research does not provide clear and/or complete answers.

When we use the term research in an academic sense it is widely accepted that we mean a process of asking questions and generating knowledge to answer these questions using rigorous accountable methods. As we have noted, reviews also share the same purposes of generating knowledge but historically we have not paid as much attention to the methods used for reviewing existing literature as we have to the methods used for primary research. Literature reviews can be used for

\footnotetext{
M. Newman $(\bowtie) \cdot$ D. Gough

Institute of Education, University College London, England, UK

e-mail: mark.newman@ucl.ac.uk

D. Gough

e-mail: david.gough@ucl.ac.uk
}

O. Zawacki-Richter et al. (eds.), Systematic Reviews in Educational Research, https://doi.org/10.1007/978-3-658-27602-7_1 
making claims about what we know and do not know about a phenomenon and also about what new research we need to undertake to address questions that are unanswered. Therefore, it seems reasonable to conclude that 'how' we conduct a review of research is important.

The increased focus on the use of research evidence to inform policy and practice decision-making in Evidence Informed Education (Hargreaves 1996; Nelson and Campbell 2017) has increased the attention given to contextual and methodological limitations of research evidence provided by single studies. Reviews of research may help address these concerns when carried on in a systematic, rigorous and transparent manner. Thus, again emphasizing the importance of 'how' reviews are completed.

The logic of systematic reviews is that reviews are a form of research and thus can be improved by using appropriate and explicit methods. As the methods of systematic review have been applied to different types of research questions, there has been an increasing plurality of types of systematic review. Thus, the term 'systematic review' is used in this chapter to refer to a family of research approaches that are a form of secondary level analysis (secondary research) that brings together the findings of primary research to answer a research question. Systematic reviews can therefore be defined as "a review of existing research using explicit, accountable rigorous research methods" (Gough et al. 2017, p. 4).

\section{Variation in Review Methods}

Reviews can address a diverse range of research questions. Consequently, as with primary research, there are many different approaches and methods that can be applied. The choices should be dictated by the review questions. These are shaped by reviewers' assumptions about the meaning of a particular research question, the approach and methods that are best used to investigate it. Attempts to classify review approaches and methods risk making hard distinctions between methods and thereby to distract from the common defining logics that these approaches often share. A useful broad distinction is between reviews that follow a broadly configurative synthesis logic and reviews that follow a broadly aggregative synthesis logic (Sandelowski et al. 2012). However, it is important to keep in mind that most reviews have elements of both (Gough et al. 2012).

Reviews that follow a broadly configurative synthesis logic approach usually investigate research questions about meaning and interpretation to explore 
and develop theory. They tend to use exploratory and iterative review methods that emerge throughout the process of the review. Studies included in the review are likely to have investigated the phenomena of interest using methods such as interviews and observations, with data in the form of text. Reviewers are usually interested in purposive variety in the identification and selection of studies. Study quality is typically considered in terms of authenticity. Synthesis consists of the deliberative configuring of data by reviewers into patterns to create a richer conceptual understanding of a phenomenon. For example, meta ethnography (Noblit and Hare 1988) uses ethnographic data analysis methods to explore and integrate the findings of previous ethnographies in order to create higher-level conceptual explanations of phenomena. There are many other review approaches that follow a broadly configurative logic (for an overview see Barnett-Page and Thomas 2009); reflecting the variety of methods used in primary research in this tradition.

Reviews that follow a broadly aggregative synthesis logic usually investigate research questions about impacts and effects. For example, systematic reviews that seek to measure the impact of an educational intervention test the hypothesis that an intervention has the impact that has been predicted. Reviews following an aggregative synthesis logic do not tend to develop theory directly; though they can contribute by testing, exploring and refining theory. Reviews following an aggregative synthesis logic tend to specify their methods in advance (a priori) and then apply them without any deviation from a protocol. Reviewers are usually concerned to identify the comprehensive set of studies that address the research question. Studies included in the review will usually seek to determine whether there is a quantitative difference in outcome between groups receiving and not receiving an intervention. Study quality assessment in reviews following an aggregative synthesis logic focusses on the minimisation of bias and thus selection pays particular attention to homogeneity between studies. Synthesis aggregates, i.e. counts and adds together, the outcomes from individual studies using, for example, statistical meta-analysis to provide a pooled summary of effect.

\section{The Systematic Review Process}

Different types of systematic review are discussed in more detail later in this chapter. The majority of systematic review types share a common set of processes. These processes can be divided into distinct but interconnected stages as illustrated in Fig. 1. Systematic reviews need to specify a research question and the methods that will be used to investigate the question. This is often written 

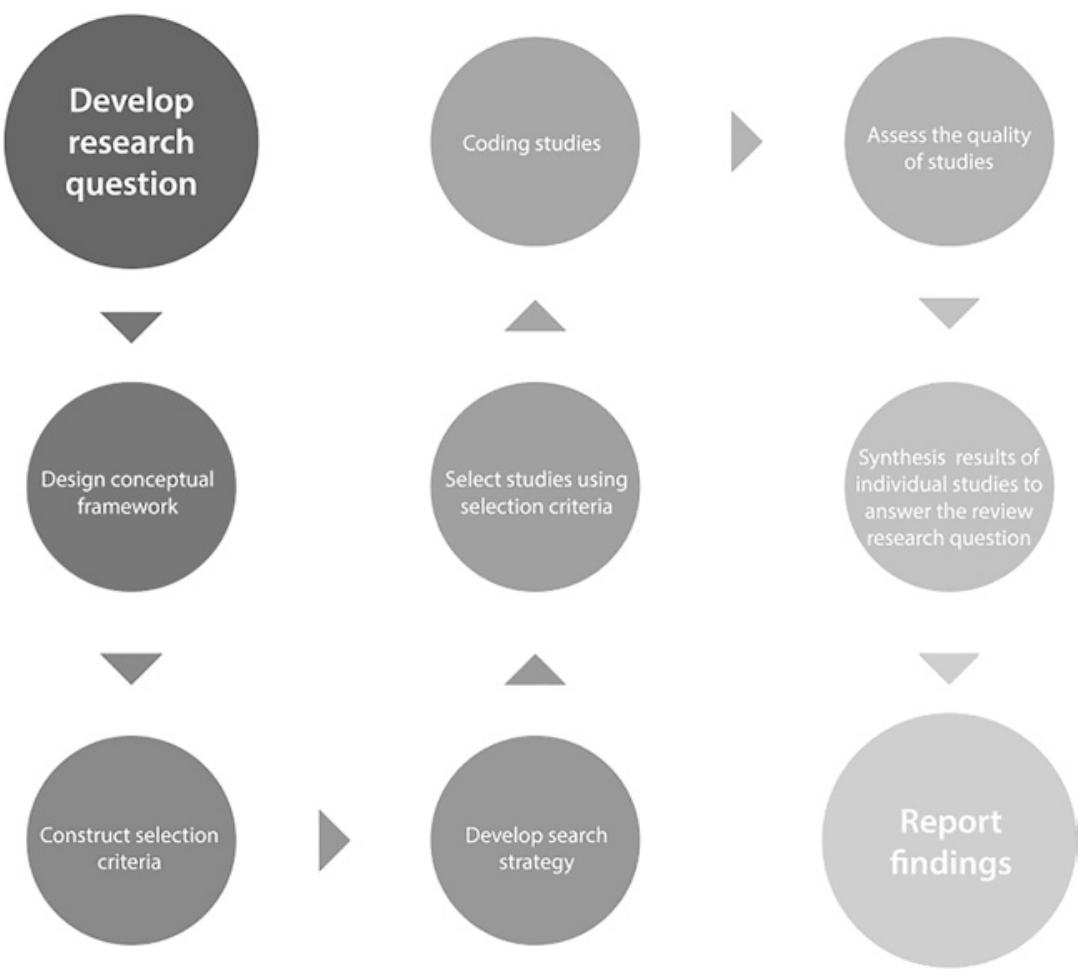

Fig. 1 The systematic review process

as a 'protocol' prior to undertaking the review. Writing a protocol or plan of the methods at the beginning of a review can be a very useful activity. It helps the review team to gain a shared understanding of the scope of the review and the methods that they will use to answer the review's questions. Different types of systematic reviews will have more or less developed protocols. For example, for systematic reviews investigating research questions about the impact of educational interventions it is argued that a detailed protocol should be fully specified prior to the commencement of the review to reduce the possibility of reviewer bias (Torgerson 2003, p. 26). For other types of systematic review, in which the research question is more exploratory, the protocol may be more flexible and/or developmental in nature. 


\subsection{Systematic Review Questions and the Conceptual Framework}

The review question gives each review its particular structure and drives key decisions about what types of studies to include; where to look for them; how to assess their quality; and how to combine their findings. Although a research question may appear to be simple, it will include many assumptions. Whether implicit or explicit, these assumptions will include: epistemological frameworks about knowledge and how we obtain it, theoretical frameworks, whether tentative or firm, about the phenomenon that is the focus of study.

Taken together, these produce a conceptual framework that shapes the research questions, choices about appropriate systematic review approach and methods. The conceptual framework may be viewed as a working hypothesis that can be developed, refined or confirmed during the course of the research. Its purpose is to explain the key issues to be studied, the constructs or variables, and the presumed relationships between them. The framework is a research tool intended to assist a researcher to develop awareness and understanding of the phenomena under scrutiny and to communicate this (Smyth 2004).

A review to investigate the impact of an educational intervention will have a conceptual framework that includes a hypothesis about a causal link between; who the review is about (the people), what the review is about (an intervention and what it is being compared with), and the possible consequences of intervention on the educational outcomes of these people. Such a review would follow a broadly aggregative synthesis logic. This is the shape of reviews of educational interventions carried out for the What Works Clearing House in the USA ${ }^{1}$ and the Education Endowment Foundation in England. ${ }^{2}$

A review to investigate meaning or understanding of a phenomenon for the purpose of building or further developing theory will still have some prior assumptions. Thus, an initial conceptual framework will contain theoretical ideas about how the phenomena of interest can be understood and some ideas justifying why a particular population and/or context is of specific interest or relevance. Such a review is likely to follow a broadly configurative logic.

\footnotetext{
${ }^{1}$ https://ies.ed.gov/ncee/wwc/

${ }^{2}$ https://educationendowmentfoundation.org.uk/evidence-summaries/teaching-learningtoolkit
} 


\subsection{Selection Criteria}

Reviewers have to make decisions about which research studies to include in their review. In order to do this systematically and transparently they develop rules about which studies can be selected into the review. Selection criteria (sometimes referred to as inclusion or exclusion criteria) create restrictions on the review. All reviews, whether systematic or not, limit in some way the studies that are considered by the review. Systematic reviews simply make these restrictions transparent and therefore consistent across studies. These selection criteria are shaped by the review question and conceptual framework. For example, a review question about the impact of homework on educational attainment would have selection criteria specifying who had to do the homework; the characteristics of the homework and the outcomes that needed to be measured. Other commonly used selection criteria include study participant characteristics; the country where the study has taken place and the language in which the study is reported. The type of research method(s) may also be used as a selection criterion but this can be controversial given the lack of consensus in education research (Newman 2008), and the inconsistent terminology used to describe education research methods.

\subsection{Developing the Search Strategy}

The search strategy is the plan for how relevant research studies will be identified. The review question and conceptual framework shape the selection criteria. The selection criteria specify the studies to be included in a review and thus are a key driver of the search strategy. A key consideration will be whether the search aims to be exhaustive i.e. aims to try and find all the primary research that has addressed the review question. Where reviews address questions about effectiveness or impact of educational interventions the issue of publication bias is a concern. Publication bias is the phenomena whereby smaller and/or studies with negative findings are less likely to be published and/or be harder to find. We may therefore inadvertently overestimate the positive effects of an educational intervention because we do not find studies with negative or smaller effects (Chow and Eckholm 2018). Where the review question is not of this type then a more specific or purposive search strategy, that may or may not evolve as the review progresses, may be appropriate. This is similar to sampling approaches in primary research. In primary research studies using aggregative approaches, such as quasiexperiments, analysis is based on the study of complete or representative samples. 
In primary research studies using configurative approaches, such as ethnography, analysis is based on examining a range of instances of the phenomena in similar or different contexts.

The search strategy will detail the sources to be searched and the way in which the sources will be searched. A list of search source types is given in Box 1 below. An exhaustive search strategy would usually include all of these sources using multiple bibliographic databases. Bibliographic databases usually index academic journals and thus are an important potential source. However, in most fields, including education, relevant research is published in a range of journals which may be indexed in different bibliographic databases and thus it may be important to search multiple bibliographic databases. Furthermore, some research is published in books and an increasing amount of research is not published in academic journals or at least may not be published there first. Thus, it is important to also consider how you will find relevant research in other sources including 'unpublished' or 'grey' literature. The Internet is a valuable resource for this purpose and should be included as a source in any search strategy.

\section{Box 1: Search Sources}

- The World Wide Web/Internet

- Google, Specialist Websites, Google Scholar, Microsoft Academic

- Bibliographic Databases

- Subject specific e.g. Education-ERIC: Education Resources Information Centre

- Generic e.g. ASSIA: Applied Social Sciences Index and Abstracts

- Handsearching of specialist journals or books

- Contacts with Experts

- Citation Checking

New, federated search engines are being developed, which search multiple sources at the same time, eliminating duplicates automatically (Tsafnat et al. 2013). Technologies, including text mining, are being used to help develop search strategies, by suggesting topics and terms on which to search-terms that reviewers may not have thought of using. Searching is also being aided by technology through the increased use (and automation) of 'citation chasing', where papers that cite, or are cited by, a relevant study are checked in case they too are relevant.

A search strategy will identify the search terms that will be used to search the bibliographic databases. Bibliographic databases usually index records 
according to their topic using 'keywords' or 'controlled terms' (categories used by the database to classify papers). A comprehensive search strategy usually involves searching both a freetext search using keywords determined by the reviewers and controlled terms. An example of a bibliographic database search is given in Box 2. This search was used in a review that aimed to find studies that investigated the impact of Youth Work on positive youth outcomes (Dickson et al. 2013). The search is built using terms for the population of interest (Youth), the intervention of interest (Youth Work) and the outcomes of Interest (Positive Development). It used both keywords and controlled terms, 'wildcards' (the *sign in this database) and the Boolean operators 'OR' and 'AND' to combine terms. This example illustrates the potential complexity of bibliographic database search strings, which will usually require a process of iterative development to finalise.

Box 2: Search string example To identify studies that address the question What is the empirical research evidence on the impact of youth work on the lives of children and young people aged 10-24 years?: CSA ERIC Database

$((\mathrm{TI}=($ adolescen* or ("young man*”) or ("young men")) or TI = (("young woman*") or ("young women") or (Young adult*”)) or TI=(("young person*") or ("young people*") or teen*) or $\mathrm{AB}=$ (adolescen* or ("young man*") or ("young men")) or $\mathrm{AB}=($ ("young woman*") or ("young women") or (Young adult*")) or $\mathrm{AB}=($ (“young person*”) or ("young people*") or teen*)) or ( $\mathrm{DE}=$ ("youth" or "adolescents" or "early adolescents" or "late adolescents" or "preadolescents"))) and $(($ TI $=(($ "positive youth development ") or ("youth development") or ("youth program*”)) or TI=(("youth club*") or ("youth work") or ("youth opportunit"”)) or $\mathrm{TI}=(($ "extended school*") or ("civic engagement") or ("positive peer culture")) or $\mathrm{TI}=($ ("informal learning") or multicomponent or ("multi-component ")) or $\mathrm{TI}=(($ "multi component") or multidimensional or ("multi-dimensional ")) or $\mathrm{TI}=($ ("multi dimensional") or empower* or asset*) or $\mathrm{TI}=$ (thriv* or ("positive development") or resilienc*) or TI=(("positive activity") or ("positive activities") or experiential) or $\mathrm{TI}=($ ("community based") or "community-based")) $\operatorname{or}(\mathrm{AB}=(($ "positive youth development ") or ("youth development") or ("youth program*")) or $\mathrm{AB}=($ ("youth club*") or ("youth work") or ("youth opportunit*")) or $\mathrm{AB}=($ ("extended school*") or ("civic engagement") or ("positive peer culture")) or $\mathrm{AB}=($ ("informal learning") or 
multicomponent or ("multi-component ")) or $\mathrm{AB}=$ ((“multi component") or multidimensional or ("multi-dimensional ")) or $\mathrm{AB}=($ ("multi dimensional") or empower* or asset*) or $\mathrm{AB}=$ (thriv* or ("positive development") or resilienc*) or $\mathrm{AB}=$ (("positive activity") or ("positive activities") or experiential) or $\mathrm{AB}=(($ (community based") or "community-based"))) or (DE="community education"))

Detailed guidance for finding effectiveness studies is available from the Campbell Collaboration (Kugley et al. 2015). Guidance for finding a broader range of studies has been produced by the EPPI-Centre (Brunton et al. 2017a).

\subsection{The Study Selection Process}

Studies identified by the search are subject to a process of checking (sometimes referred to as screening) to ensure they meet the selection criteria. This is usually done in two stages whereby titles and abstracts are checked first to determine whether the study is likely to be relevant and then a full copy of the paper is acquired to complete the screening exercise. The process of finding studies is not efficient. Searching bibliographic databases, for example, leads to many irrelevant studies being found which then have to be checked manually one by one to find the few relevant studies. There is increasing use of specialised software to support and in some cases, automate the selection process. Text mining, for example, can assist in selecting studies for a review (Brunton et al. 2017b). A typical text mining or machine learning process might involve humans undertaking some screening, the results of which are used to train the computer software to learn the difference between included and excluded studies and thus be able to indicate which of the remaining studies are more likely to be relevant. Such automated support may result in some errors in selection, but this may be less than the human error in manual selection (O'Mara-Eves et al. 2015).

\subsection{Coding Studies}

Once relevant studies have been selected, reviewers need to systematically identify and record the information from the study that will be used to answer the review question. This information includes the characteristics of the studies, 
including details of the participants and contexts. The coding describes: (i) details of the studies to enable mapping of what research has been undertaken; (ii) how the research was undertaken to allow assessment of the quality and relevance of the studies in addressing the review question; (iii) the results of each study so that these can be synthesised to answer the review question.

The information is usually coded into a data collection system using some kind of technology that facilitates information storage and analysis (Brunton et al. 2017b) such as the EPPI-Centre's bespoke systematic review software EPPI Reviewer. ${ }^{3}$ Decisions about which information to record will be made by the review team based on the review question and conceptual framework. For example, a systematic review about the relationship between school size and student outcomes collected data from the primary studies about each schools funding, students, teachers and school organisational structure as well as about the research methods used in the study (Newman et al. 2006). The information coded about the methods used in the research will vary depending on the type of research included and the approach that will be used to assess the quality and relevance of the studies (see the next section for further discussion of this point).

Similarly, the information recorded as 'results' of the individual studies will vary depending on the type of research that has been included and the approach to synthesis that will be used. Studies investigating the impact of educational interventions using statistical meta-analysis as a synthesis technique will require all of the data necessary to calculate effect sizes to be recorded from each study (see the section on synthesis below for further detail on this point). However, even in this type of study there will be multiple data that can be considered to be 'results' and so which data needs to be recorded from studies will need to be carefully specified so that recording is consistent across studies

\subsection{Appraising the Quality of Studies}

Methods are reinvented every time they are used to accommodate the real world of research practice (Sandelowski et al. 2012). The researcher undertaking a primary research study has attempted to design and execute a study that addresses the research question as rigorously as possible within the parameters of their

${ }^{3}$ https://eppi.ioe.ac.uk/cms/Default.aspx $?$ tabid=2914 
resources, understanding, and context. Given the complexity of this task, the contested views about research methods and the inconsistency of research terminology, reviewers will need to make their own judgements about the quality of the any individual piece of research included in their review. From this perspective, it is evident that using a simple criteria, such as 'published in a peer reviewed journal' as a sole indicator of quality, is not likely to be an adequate basis for considering the quality and relevance of a study for a particular systematic review.

In the context of systematic reviews this assessment of quality is often referred to as Critical Appraisal (Petticrew and Roberts 2005). There is considerable variation in what is done during critical appraisal: which dimensions of study design and methods are considered; the particular issues that are considered under each dimension; the criteria used to make judgements about these issues and the cut off points used for these criteria (Oancea and Furlong 2007). There is also variation in whether the quality assessment judgement is used for excluding studies or weighting them in analysis and when in the process judgements are made.

There are broadly three elements that are considered in critical appraisal: the appropriateness of the study design in the context of the review question, the quality of the execution of the study methods and the study's relevance to the review question (Gough 2007). Distinguishing study design from execution recognises that whilst a particular design may be viewed as more appropriate for a study it also needs to be well executed to achieve the rigour or trustworthiness attributed to the design. Study relevance is achieved by the review selection criteria but assessing the degree of relevance recognises that some studies may be less relevant than others due to differences in, for example, the characteristics of the settings or the ways that variables are measured.

The assessment of study quality is a contested and much debated issue in all research fields. Many published scales are available for assessing study quality. Each incorporates criteria relevant to the research design being evaluated. Quality scales for studies investigating the impact of interventions using (quasi) experimental research designs tend to emphasis establishing descriptive causality through minimising the effects of bias (for detailed discussion of issues associated with assessing study quality in this tradition see Waddington et al. 2017). Quality scales for appraising qualitative research tend to focus on the extent to which the study is authentic in reflecting on the meaning of the data (for detailed discussion of the issues associated with assessing study quality in this tradition see Carroll and Booth 2015). 


\subsection{Synthesis}

A synthesis is more than a list of findings from the included studies. It is an attempt to integrate the information from the individual studies to produce a 'better' answer to the review question than is provided by the individual studies. Each stage of the review contributes toward the synthesis and so decisions made in earlier stages of the review shape the possibilities for synthesis. All types of synthesis involve some kind of data transformation that is achieved through common analytic steps: searching for patterns in data; Checking the quality of the synthesis; Integrating data to answer the review question (Thomas et al. 2012). The techniques used to achieve these vary for different types of synthesis and may appear more or less evident as distinct steps.

Statistical meta-analysis is an aggregative synthesis approach in which the outcome results from individual studies are transformed into a standardized, scale free, common metric and combined to produce a single pooled weighted estimate of effect size and direction. There are a number of different metrics of effect size, selection of which is principally determined by the structure of outcome data in the primary studies as either continuous or dichotomous. Outcome data with a dichotomous structure can be transformed into Odds Ratios (OR), Absolute Risk Ratios (ARR) or Relative Risk Ratios (RRR) (for detailed discussion of dichotomous outcome effect sizes see Altman 1991). More commonly seen in education research, outcome data with a continuous structure can be translated into Standardised Mean Differences (SMD) (Fitz-Gibbon 1984). At its most straightforward effect size calculation is simple arithmetic. However given the variety of analysis methods used and the inconsistency of reporting in primary studies it is also possible to calculate effect sizes using more complex transformation formulae (for detailed instructions on calculating effect sizes from a wide variety of data presentations see Lipsey and Wilson 2000).

The combination of individual effect sizes uses statistical procedures in which weighting is given to the effect sizes from the individual studies based on different assumptions about the causes of variance and this requires the use of statistical software. Statistical measures of heterogeneity produced as part of the meta-analysis are used to both explore patterns in the data and to assess the quality of the synthesis (Thomas et al. 2017a).

In configurative synthesis the different kinds of text about individual studies and their results are meshed and linked to produce patterns in the data, explore different configurations of the data and to produce new synthetic accounts of the phenomena under investigation. The results from the individual studies are 
translated into and across each other, searching for areas of commonality and refutation. The specific techniques used are derived from the techniques used in primary research in this tradition. They include reading and re-reading, descriptive and analytical coding, the development of themes, constant comparison, negative case analysis and iteration with theory (Thomas et al. 2017b).

\section{$4 \quad$ Variation in Review Structures}

All research requires time and resources and systematic reviews are no exception. There is always concern to use resources as efficiently as possible. For these reasons there is a continuing interest in how reviews can be carried out more quickly using fewer resources. A key issue is the basis for considering a review to be systematic. Any definitions are clearly open to interpretation. Any review can be argued to be insufficiently rigorous and explicit in method in any part of the review process. To assist reviewers in being rigorous, reporting standards and appraisal tools are being developed to assess what is required in different types of review (Lockwood and Geum Oh 2017) but these are also the subject of debate and disagreement.

In addition to the term 'systematic review' other terms are used to denote the outputs of systematic review processes. Some use the term 'scoping review' for a quick review that does not follow a fully systematic process. This term is also used by others (for example, Arksey and O'Malley 2005) to denote 'systematic maps' that describe the nature of a research field rather than synthesise findings. A 'quick review' type of scoping review may also be used as preliminary work to inform a fuller systematic review. Another term used is 'rapid evidence assessment'. This term is usually used when systematic review needs to be undertaken quickly and in order to do this the methods of review are employed in a more minimal than usual way. For example, by more limited searching. Where such 'shortcuts' are taken there may be some loss of rigour, breadth and/or depth (Abrami et al. 2010; Thomas et al. 2013).

Another development has seen the emergence of the concept of 'living reviews', which do not have a fixed end point but are updated as new relevant primary studies are produced. Many review teams hope that their review will be updated over time, but what is different about living reviews is that it is built into the system from the start as an on-going developmental process. This means that the distribution of review effort is quite different to a standard systematic review, being a continuous lower-level effort spread over a longer time period, rather than the shorter bursts of intensive effort that characterise a review with periodic updates (Elliott et al. 2014). 


\subsection{Systematic Maps and Syntheses}

One potentially useful aspect of reviewing the literature systematically is that it is possible to gain an understanding of the breadth, purpose and extent of research activity about a phenomenon. Reviewers can be more informed about how research on the phenomenon has been constructed and focused. This type of reviewing is known as 'mapping' (see for example, Peersman 1996; Gough et al. 2003). The aspects of the studies that are described in a map will depend on what is of most interest to those undertaking the review. This might include information such as topic focus, conceptual approach, method, aims, authors, location and context. The boundaries and purposes of a map are determined by decisions made regarding the breadth and depth of the review, which are informed by and reflected in the review question and selection criteria.

Maps can also be a useful stage in a systematic review where study findings are synthesised as well. Most synthesis reviews implicitly or explicitly include some sort of map in that they describe the nature of the relevant studies that they have identified. An explicit map is likely to be more detailed and can be used to inform the synthesis stage of a review. It can provide more information on the individual and grouped studies and thus also provide insights to help inform choices about the focus and strategy to be used in a subsequent synthesis.

\subsection{Mixed Methods, Mixed Research Synthesis Reviews}

Where studies included in a review consist of more than one type of study design, there may also be different types of data. These different types of studies and data can be analysed together in an integrated design or segregated and analysed separately (Sandelowski et al. 2012). In a segregated design, two or more separate sub-reviews are undertaken simultaneously to address different aspects of the same review question and are then compared with one another.

Such 'mixed methods' and 'multiple component' reviews are usually necessary when there are multiple layers of review question or when one study design alone would be insufficient to answer the question(s) adequately. The reviews are usually required, to have both breadth and depth. In doing so they can investigate a greater extent of the research problem than would be the case in a more focussed single method review. As they are major undertakings, containing what would normally be considered the work of multiple systematic reviews, they are demanding of time and resources and cannot be conducted quickly. 


\subsection{Reviews of Reviews}

Systematic reviews of primary research are secondary levels of research analysis. A review of reviews (sometimes called 'overviews' or 'umbrella' reviews) is a tertiary level of analysis. It is a systematic map and/or synthesis of previous reviews. The 'data' for reviews of reviews are previous reviews rather than primary research studies (see for example Newman et al. (2018). Some review of reviews use previous reviews to combine both primary research data and synthesis data. It is also possible to have hybrid review models consisting of a review of reviews and then new systematic reviews of primary studies to fill in gaps in coverage where there is not an existing review (Caird et al. 2015). Reviews of reviews can be an efficient method for examining previous research. However, this approach is still comparatively novel and questions remain about the appropriate methodology. For example, care is required when assessing the way in which the source systematic reviews identified and selected data for inclusion, assessed study quality and to assess the overlap between the individual reviews (Aromataris et al. 2015).

\section{$5 \quad$ Other Types of Research Based Review Structures}

This chapter so far has presented a process or method that is shared by many different approaches within the family of systematic review approaches, notwithstanding differences in review question and types of study that are included as evidence. This is a helpful heuristic device for designing and reading systematic reviews. However, it is the case that there are some review approaches that also claim to use a research based review approach but that do not claim to be systematic reviews and or do not conform with the description of processes that we have given above at all or in part at least.

\subsection{Realist Synthesis Reviews}

Realist synthesis is a member of the theory-based school of evaluation (Pawson 2002). This means that it is underpinned by a 'generative' understanding of causation, which holds that, to infer a causal outcome/relationship between an intervention (e.g. a training programme) and an outcome $(\mathrm{O})$ of interest (e.g. unemployment), one needs to understand the underlying mechanisms $(\mathrm{M})$ that connect them and the context (C) in which the relationship occurs (e.g. the characteristics of both the subjects and the programme locality). The interest of this 
approach (and also of other theory driven reviews) is not simply which interventions work, but which mechanisms work in which context. Rather than identifying replications of the same intervention, the reviews adopt an investigative stance and identify different contexts within which the same underlying mechanism is operating.

Realist synthesis is concerned with hypothesising, testing and refining such context-mechanism-outcome (CMO) configurations. Based on the premise that programmes work in limited circumstances, the discovery of these conditions becomes the main task of realist synthesis. The overall intention is to first create an abstract model (based on the CMO configurations) of how and why programmes work and then to test this empirically against the research evidence. Thus, the unit of analysis in a realist synthesis is the programme mechanism, and this mechanism is the basis of the search. This means that a realist synthesis aims to identify different situations in which the same programme mechanism has been attempted. Integrative Reviewing, which is aligned to the Critical Realist tradition, follows a similar approach and methods (Jones-Devitt et al. 2017).

\subsection{Critical Interpretive Synthesis (CIS)}

Critical Interpretive Synthesis (CIS) (Dixon-Woods et al. 2006) takes a position that there is an explicit role for the 'authorial' (reviewer's) voice in the review. The approach is derived from a distinctive tradition within qualitative enquiry and draws on some of the tenets of grounded theory in order to support explicitly the process of theory generation. In practice, this is operationalised in its inductive approach to searching and to developing the review question as part of the review process, its rejection of a 'staged' approach to reviewing and embracing the concept of theoretical sampling in order to select studies for inclusion. When assessing the quality of studies CIS prioritises relevance and theoretical contribution over research methods. In particular, a critical approach to reading the literature is fundamental in terms of contextualising findings within an analysis of the research traditions or theoretical assumptions of the studies included.

\subsection{Meta-Narrative Reviews}

Meta-narrative reviews, like critical interpretative synthesis, place centre-stage the importance of understanding the literature critically and understanding differences between research studies as possibly being due to differences between their underlying research traditions (Greenhalgh et al. 2005). This means that 
each piece of research is located (and, when appropriate, aggregated) within its own research tradition and the development of knowledge is traced (configured) through time and across paradigms. Rather than the individual study, the 'unit of analysis' is the unfolding 'storyline' of a research tradition over time' (Greenhalgh et al. 2005).

\section{Conclusions}

This chapter has briefly described the methods, application and different perspectives in the family of systematic review approaches. We have emphasized the many ways in which systematic reviews can vary. This variation links to different research aims and review questions. But also to the different assumptions made by reviewers. These assumptions derive from different understandings of research paradigms and methods and from the personal, political perspectives they bring to their research practice. Although there are a variety of possible types of systematic reviews, a distinction in the extent that reviews follow an aggregative or configuring synthesis logic is useful for understanding variations in review approaches and methods. It can help clarify the ways in which reviews vary in the nature of their questions, concepts, procedures, inference and impact. Systematic review approaches continue to evolve alongside critical debate about the merits of various review approaches (systematic or otherwise). So there are many ways in which educational researchers can use and engage with systematic review methods to increase knowledge and understanding in the field of education.

\section{References}

Abrami, P. C. Borokhovski, E. Bernard, R. M. Wade, CA. Tamim, R. Persson, T. Bethel, E. C. Hanz, K. \& Surkes, M. A. (2010). Issues in conducting and disseminating brief reviews of evidence. Evidence \& Policy, 6(3), 371-389.

Altman, D.G. (1991) Practical statistics for medical research. London: Chapman and Hall.

Arksey, H. \& O’Malley, L. (2005). Scoping studies: towards a methodological framework, International Journal of Social Research Methodology, 8(1), 19-32,

Aromataris, E. Fernandez, R. Godfrey, C. Holly, C. Khalil, H. Tungpunkom, P. (2015). Summarizing systematic reviews: methodological development, conduct and reporting of an umbrella review approach. International Journal of Evidence-Based Healthcare, 13.

Barnett-Page, E. \& Thomas, J. (2009). Methods for the synthesis of qualitative research: A critical review, BMC Medical Research Methodology, 9(59), https://doi. org/10.1186/1471-2288-9-59. 
Brunton, G., Stansfield, C., Caird, J. \& Thomas, J. (2017a). Finding relevant studies. In D. Gough, S. Oliver \& J. Thomas (Eds.), An introduction to systematic reviews (2nd edition, pp. 93-132). London: Sage.

Brunton, J., Graziosi, S., \& Thomas, J. (2017b). Tools and techniques for information management. In D. Gough, S. Oliver \& J. Thomas (Eds.), An introduction to systematic reviews (2nd edition, pp. 154-180), London: Sage.

Carroll, C. \& Booth, A. (2015). Quality assessment of qualitative evidence for systematic review and synthesis: Is it meaningful, and if so, how should it be performed? Research Synthesis Methods 6(2), 149-154.

Caird, J. Sutcliffe, K. Kwan, I. Dickson, K. \& Thomas, J. (2015). Mediating policy-relevant evidence at speed: are systematic reviews of systematic reviews a useful approach? Evidence \& Policy, 11(1), 81-97.

Chow, J. \& Eckholm, E. (2018). Do published studies yield larger effect sizes than unpublished studies in education and special education? A meta-review. Educational Psychology Review 30(3), 727-744.

Dickson, K., Vigurs, C. \& Newman, M. (2013). Youth work a systematic map of the literature. Dublin: Dept of Government Affairs.

Dixon-Woods, M., Cavers, D., Agarwa, S., Annandale, E. Arthur, A., Harvey, J., Hsu, R., Katbamna, S., Olsen, R., Smith, L., Riley R., \& Sutton, A. J. (2006). Conducting a critical interpretive synthesis of the literature on access to healthcare by vulnerable groups. BMC Medical Research Methodology 6:35 https://doi.org/10.1186/1471-2288-6-35.

Elliott, J. H., Turner, T., Clavisi, O., Thomas, J., Higgins, J. P. T., Mavergames, C. \& Gruen, R. L. (2014). Living systematic reviews: an emerging opportunity to narrow the evidence-practice gap. PLoS Medicine, 11(2): e1001603. http://doi.org/10.1371/journal. pmed.1001603.

Fitz-Gibbon, C.T. (1984) Meta-analysis: an explication. British Educational Research Journal, 10(2), 135-144.

Gough, D. (2007). Weight of evidence: a framework for the appraisal of the quality and relevance of evidence. Research Papers in Education, 22(2), 213-228.

Gough, D., Thomas, J. \& Oliver, S. (2012). Clarifying differences between review designs and methods. Systematic Reviews, 1(28).

Gough, D., Kiwan, D., Sutcliffe, K., Simpson, D. \& Houghton, N. (2003). A systematic map and synthesis review of the effectiveness of personal development planning for improving student learning. In: Research Evidence in Education Library. London: EPPI-Centre, Social Science Research Unit, Institute of Education, University of London.

Gough, D., Oliver, S. \& Thomas, J. (2017). Introducing systematic reviews. In D. Gough, S. Oliver \& J. Thomas (Eds.), An introduction to systematic reviews (2nd edition, pp. 1-18). London: Sage.

Greenhalgh, T., Robert, G., Macfarlane, F., Bate, P., Kyriakidou, O. \& Peacock, R. (2005). Storylines of research in diffusion of innovation: A meta-narrative approach to systematic review. Social Science \& Medicine, 61(2), 417-430.

Hargreaves, D. (1996). Teaching as a research based profession: possibilities and prospects. Teacher Training Agency Annual Lecture. Retrieved from https://eppi.ioe.ac.uk/ cms/Default.aspx ?tabid=2082. 
Hart, C. (2018). Doing a literature review: releasing the research imagination. London. SAGE.

Jones-Devitt, S. Austen, L. \& Parkin H. J. (2017). Integrative reviewing for exploring complex phenomena. Social Research Update. Issue 66.

Kugley, S., Wade, A., Thomas, J., Mahood, Q., Klint Jørgensen, A. M., Hammerstrøm, K., \& Sathe, N. (2015). Searching for studies: A guide to information retrieval for Campbell Systematic Reviews. Campbell Method Guides 2016:1 (http://www.campbellcollaboration.org/images/Campbell_Methods_Guides_Information_Retrieval.pdf).

Lipsey, M.W., \& Wilson, D. B. (2000). Practical meta-analysis. Thousand Oaks: Sage.

Lockwood, C. \& Geum Oh, E. (2017). Systematic reviews: guidelines, tools and checklists for authors. Nursing \& Health Sciences, 19, 273-277.

Nelson, J. \& Campbell, C. (2017). Evidence-informed practice in education: meanings and applications. Educational Research, 59(2), 127-135.

Newman, M., Garrett, Z., Elbourne, D., Bradley, S., Nodenc, P., Taylor, J. \& West, A. (2006). Does secondary school size make a difference? A systematic review. Educational Research Review, 1(1), 41-60.

Newman, M. (2008). High quality randomized experimental research evidence: Necessary but not sufficient for effective education policy. Psychology of Education Review, 32(2), 14-16.

Newman, M., Reeves, S. \& Fletcher, S. (2018). A critical analysis of evidence about the impacts of faculty development in systematic reviews: a systematic rapid evidence assessment. Journal of Continuing Education in the Health Professions, 38(2), 137144.

Noblit, G. W. \& Hare, R. D. (1988). Meta-ethnography: Synthesizing qualitative studies. Newbury Park, CA: Sage.

Oancea, A. \& Furlong, J. (2007). Expressions of excellence and the assessment of applied and practice-based research. Research Papers in Education 22.

O’Mara-Eves, A., Thomas, J., McNaught, J., Miwa, M., \& Ananiadou, S. (2015). Using text mining for study identification in systematic reviews: a systematic review of current approaches. Systematic Reviews 4(1): 5. https://doi.org/10.1186/2046-4053-4-5.

Pawson, R. (2002). Evidence-based policy: the promise of "Realist Synthesis". Evaluation, $8(3), 340-358$.

Peersman, G. (1996). A descriptive mapping of health promotion studies in young people, EPPI Research Report. London: EPPI-Centre, Social Science Research Unit, Institute of Education, University of London.

Petticrew, M. \& Roberts, H. (2005). Systematic reviews in the social sciences: a practical guide. London: Wiley.

Sandelowski, M., Voils, C. I., Leeman, J. \& Crandell, J. L. (2012). Mapping the mixed methods-mixed research synthesis terrain. Journal of Mixed Methods Research, 6(4), 317-331.

Smyth, R. (2004). Exploring the usefulness of a conceptual framework as a research tool: A researcher's reflections. Issues in Educational Research, 14.

Thomas, J., Harden, A. \& Newman, M. (2012). Synthesis: combining results systematically and appropriately. In D. Gough, S. Oliver \& J. Thomas (Eds.), An introduction to systematic reviews (pp. 66-82). London: Sage. 
Thomas, J., Newman, M. \& Oliver, S. (2013). Rapid evidence assessments of research to inform social policy: taking stock and moving forward. Evidence and Policy, 9(1), $5-27$.

Thomas, J., O’Mara-Eves, A., Kneale, D. \& Shemilt, I. (2017a). Synthesis methods for combining and configuring quantitative data. In D. Gough, S. Oliver \& J. Thomas (Eds.), An Introduction to Systematic Reviews (2nd edition, pp. 211-250). London: Sage.

Thomas, J., O'Mara-Eves, A., Harden, A. \& Newman, M. (2017b). Synthesis methods for combining and configuring textual or mixed methods data. In D. Gough, S. Oliver \& J. Thomas (Eds.), An introduction to systematic reviews (2nd edition, pp. 181-211), London: Sage.

Tsafnat, G., Dunn, A. G., Glasziou, P. \& Coiera, E. (2013). The automation of systematic reviews. British Medical Journal, 345(7891), doi.org/10.1136/bmj.f139.

Torgerson, C. (2003). Systematic reviews. London. Continuum.

Waddington, H., Aloe, A. M., Becker, B. J., Djimeu, E. W., Hombrados, J. G., Tugwell, P., Wells, G. \& Reeves, B. (2017). Quasi-experimental study designs series-paper 6: risk of bias assessment. Journal of Clinical Epidemiology, 89, 43-52.

Open Access This chapter is licensed under the terms of the Creative Commons Attribution 4.0 International License (http://creativecommons.org/licenses/by/4.0/), which permits use, sharing, adaptation, distribution and reproduction in any medium or format, as long as you give appropriate credit to the original author(s) and the source, provide a link to the Creative Commons license and indicate if changes were made.

The images or other third party material in this chapter are included in the chapter's Creative Commons license, unless indicated otherwise in a credit line to the material. If material is not included in the chapter's Creative Commons license and your intended use is not permitted by statutory regulation or exceeds the permitted use, you will need to obtain permission directly from the copyright holder.

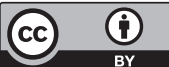

